\title{
MATHEMATICS APPLIED IN FLUID MOTION
}

\author{
BY
}

J. T. STUART

Dept. of Mathematics, Imperial College of Science, Technology and Medicine, London SW7 2BZ, UK

\begin{abstract}
Over many decades, indeed for more than a century, fluid dynamics has been the subject of many beautiful experiments and has been a proving ground for a wealth of mathematical theories, both linear and nonlinear.

We can ask: What was an outstanding problem for Osborne Reynolds (1883) over one hundred years ago? And what is an outstanding problem even now?

One answer is turbulence! And this answer is valid in spite of the many advances in experiment, theory and computation over the intervening decades.

We would be pleased to know about the structure of turbulence, and of its occurrence intermittently in an otherwise laminar-flow environment. In this connection we can and do refer to spots, puffs and slugs of turbulence. This paper focusses attention on our present knowledge of such phenomena, and on the challenges posed by our lack of understanding of many aspects of turbulent processes.
\end{abstract}

1. Introduction. We wish to concentrate on two important prototype experiments: one is the flow in a long circular pipe while the other is the flow over a flat plate.

For the flow in a pipe of radius $a$ and maximum velocity $U_{0}$, it is known that the Hagen-Poiseuille laminar flow, $u$, is given non-dimensionally by $u=1-r^{2}$, where $r$ is the non-dimensional radial coordinate. It is known (Ekman 1910; Taylor, see Rosenhead 1963) that such a laminar flow can be maintained even at a Reynolds number $\left(R=U_{0} a / \nu\right.$, where $\nu$ is the kinematic viscosity) as high as 44000. Moreover, Reynolds (1883) observed flashes of disordered motion in a background of smooth, ordered flow. Did such flashes arise from conditions at the entry of the pipe, or from natural instabilities of the flow, or from some other cause? Such questions have been with us for more than a century, and our understanding is still imperfect to say the least.

Instability theories have been developed for laminar flow in a circular pipe, although the outcome is not satisfactory. The flow near to the inlet of the pipe, where there is a region of sharp change, a boundary layer, on the wall, is known to be unstable in the sense that linear eigenmode perturbations can amplify, as shown by Tatsumi (1952). Further along the pipe, however, where the laminar flow is fully developed, all existing

Received March 26, 1998.

1991 Mathematics Subject Classification. Primary 76E30, 76E05, 76F10.

E-mail address: t.stuart@ic.ac.uk 
theoretical work on linear eigenmodes suggests that they decay; but there is no accepted proof of this. Furthermore, it is natural to inquire about the influence of nonlinearity on instabilities of laminar flow: but in spite of some interesting pieces of mathematical work, there is no satisfactory nonlinear theory. Thus our understanding is not good.

In experiment and observation, Wygnanski and others $(1973,1975)$ have shown the existence of "puffs" and "slugs" of turbulence in flows, these entities being characterised by rather sharp interfaces between turbulent (random, chaotic) and laminar regions of flow.

What do we know of sharp interfaces? Are there related phenomena elsewhere, as in shock waves, hydraulic jumps, and vortex breakdown? Or is the laminar turbulent sharp interface rather special, requiring its own treatment? Inquiries are still in the early stages.

From the point of view of modern technology, much attention has been focussed on flow in the boundary-layer of the flow of air over, for example, an aircraft wing. Work by Prandtl, Heisenberg, Tollmien, Lin, Meksyn, and many others (see the book edited by Rosenhead 1963) showed the possibility of instability waves evolving in a boundary-layer flow; and then in the early 1940s Schubauer and Skramstad (1947) showed the existence of such waves in their beautiful experiments done in Washington. Later work by Schubauer and Klebanoff (1955) showed experimentally that the instability (vorticity) waves often lead to "spots" of turbulence which occur intermittently and are characterised by sharp interfaces. So here also there arises the question of sharp interfaces between turbulent and laminar regions of flow. There is a real need to have a better theoretical understanding of such interfaces. Some possibly-relevant factors will be discussed later in this paper.

2. Equations of motion. As is well known, there are two ways to describe fluid motion, the Eulerian and the Lagrangian modes of description.

In the Eulerian scheme we consider a particle at a vector position $\mathbf{x}$ at time $t$. Then the density $\rho$, the pressure $p$, the velocity $\mathbf{u}$, and the vorticity $\boldsymbol{\omega}$ are considered to be functions of $\mathbf{x}$ and $t$. Crucially the time-dependent vector velocity is defined at a point. Then the equations of motion are

$$
\begin{gathered}
\rho \frac{d \mathbf{u}}{d t}=\rho\left(\frac{\partial \mathbf{u}}{\partial t}+\mathbf{u} \cdot \nabla \mathbf{u}\right)=-\nabla p+\text { viscous terms } \\
\frac{\partial \rho}{\partial t}+\nabla \cdot(\rho \mathbf{u})=0 \\
\boldsymbol{\omega}=\operatorname{curl} \mathbf{u}
\end{gathered}
$$

and, if the fluid is barotropic,

$$
p=f(\rho) .
$$

The viscous terms are characterised by

$$
\mu\left(\nabla^{2} \mathbf{u}+\text { other terms }\right)
$$

where $\mu$ is the coefficient of viscosity. 
The vorticity equation can be obtained by elimination of $p$ from (2.1); for the incompressible case of constant density $(\rho)$ the equation is

$$
\frac{\partial \boldsymbol{\omega}}{\partial t}+\mathbf{u} \cdot \nabla \omega=\boldsymbol{\omega} \cdot \nabla \mathbf{u}+\mu \nabla^{2} \omega
$$

but there are extra terms if compressibility is present.

In the Lagrangian scheme the position of the particle $(\mathbf{x})$ is considered to be the dependent variable, being a function of its initial position a at $t=0$ and of the current time $t$. The velocity $\mathbf{u}$ and acceleration are given by

$$
\mathbf{u}=\frac{\partial \mathbf{x}(\mathbf{a}, t)}{\partial t}, \quad \frac{\partial \mathbf{u}}{\partial t}=\frac{\partial^{2} \mathbf{x}(\mathbf{a}, t)}{\partial t^{2}} .
$$

For the equation of motion, (2.1) is replaced by

$$
\rho\left(\frac{\partial^{2} \mathbf{x}}{\partial t^{2}}\right)=-\nabla p+\text { viscous terms, }
$$

while the equation of continuity (2.2) becomes

$$
\begin{gathered}
\rho J=\rho_{0}, \\
J=\left|\frac{\partial x_{i}}{\partial a_{j}}\right| .
\end{gathered}
$$

Here $\rho_{0}(\mathbf{a})$ is the density at $t=0$. Moreover, the gradient operator is an operator on $\mathbf{x}$, although $p$ is a function of $\mathbf{a}$ and $t$.

3. Interface phenomena. Turbulent spots were first described in some detail by Emmons and Bryson (1951), who observed the phenomenon in the flow of a layer of water over a flat surface. Later Schubauer and Klebanoff (1955) took up the study of spots for air flow over a flat plate with a flow speed of about $10 \mathrm{~m} / \mathrm{s}$. A spot was initiated by an electric spark at $0.75 \mathrm{~m}$ from the leading edge of the plate; then measurements were made at locations $0.8 \mathrm{~m}$ and $1.5 \mathrm{~m}$ from the spark. Typical oscillogram records, which indicate the velocity response, are shown in their paper. A sharp rise in the signal is followed by oscillations and then a slow decay.

Detailed observations led Schubauer and Klebanoff to the following picture. The maximum speed of any part of the spot is that of the free stream $U_{0}=10 \mathrm{~m} / \mathrm{s}$. But nearer to the surface the velocity is typically $0.5 U_{0}$ at the rear and $0.88 U_{0}$ at the front of the spot, at least in their experiments. The angle of spread of the turbulent spot is about $22^{\circ}$, which is known to be quite typical. Details of the internal structure have been given by Cantwell, Coles, and Dimotakis (1978), while numerical simulations are due to Henningsen, Spalart, and Kim (1987). The experimental and theoretical work of Gaster (1982) is also relevant.

Thus there are real questions to ask of the origin of turbulent spots, of the structure of the interfaces, of the structure of the interior and of their angle of spread. Relevant factors can be surface imperfections, free-stream imperfections, the electric spark as a generator and, most particularly, the vorticity field. Compressibility may also be a factor in regions of rapid changes. In the following sections we shall evaluate some of these possible factors. 
4. The role of vorticity. One mechanism for the origin and development of turbulent spots in a boundary layer lies with the role played by vorticity.

In a plane, two-dimensional, flow the vorticity is orthogonal to the velocity field. Furthermore, we know that if the viscosity $(\mu)$ is zero, the vorticity is then simply convected by the flow field: this is shown clearly by (2.6) since $\mu \equiv 0$ and $\boldsymbol{\omega} \cdot \nabla \mathbf{u} \equiv 0$ (from orthogonality of the vorticity and velocity fields).

On the other hand, a general three-dimensional flow behaves quite differently even if $\mu \equiv 0$. The difference lies with the term $\boldsymbol{\omega} \cdot \nabla \mathbf{u}$, which is nonzero in general: it represents the increase or decrease of vorticity associated with the stretching or tilting of vortex filaments, by gyroscopic effects in fact. We can illustrate these phenomena as follows.

Suppose we have an incompressible, inviscid flow in which both the pressure and velocity fields are independent of $x$. Then the first component of Eq. (2.1) is

$$
\frac{\partial u}{\partial t}+v \frac{\partial u}{\partial y}+w \frac{\partial u}{\partial z}=0
$$

We can suppose that $v, w$, and $p$ are given by the second and third components of (2.1) or are supplied from experimental observation. Then (4.1) is a linear equation for the velocity component $u$.

Associated with (4.1) is a vorticity equation. If we denote $\omega$ by $(\xi, \eta, \zeta)$ then the third component of $(2.6)$ is

$$
\frac{\partial \zeta}{\partial t}+v \frac{\partial \zeta}{\partial y}+w \frac{\partial \zeta}{\partial z}=\eta \frac{\partial w}{\partial y}+\zeta \frac{\partial w}{\partial z}
$$

This equation represents a $y$ derivative of (4.1) since

$$
\boldsymbol{\omega} \equiv(\xi, \eta, \zeta)=\left(\frac{\partial w}{\partial y}-\frac{\partial v}{\partial z}, \frac{\partial u}{\partial z},-\frac{\partial u}{\partial y}\right)
$$

and (2.2) can be applied with $\rho=$ constant. On the right-hand side of (4.2), the first term represents the tilting of the vorticity component $\eta$ by the shear $\partial w / \partial y$; the second term represents the stretching of the component $\zeta$ by the strain $\partial w / \partial z$. It is known that these processes are fundamental in complex fluid motions, not least in anything associated with turbulence.

Let us now return to (4.1) with $v$ and $w$ known as functions of $y$ and $z$. Then $u$ can be obtained subject to an initial condition at $t=0$. Calculation shows that a typical boundary layer velocity distribution for $u$ at $t=0$ evolves for $t>0$ with a more convoluted shape with a developing sharp gradient at some value of $y$. This sharp gradient in the velocity component $u$ represents a large spanwise $(z)$ component of vorticity, as is shown by (4.3). At $z$ locations where there is a symmetry in the $z$ direction, $\partial u / \partial z$ is identically zero: then the strong increase in vorticity is brought about by the second term on the right-hand side of (4.2), which represents vortex stretching, as has been mentioned already.

It was observed by Klebanoff, Tidstrom, and Sargent (1962) that at locations where the vorticity is strongly enhanced, as is the case in the example described, then an embryonic turbulent spot of vorticity is often developed. This then is one possible source of turbulent spots, although the details are far from completely understood. 
Given that turbulent spots can arise we can still ask, and need to understand, the way in which an interface between a laminar, or nearly-laminar, region and a turbulent region can be sustained. How is random vorticity produced or transferred across an interface? How relevant are the processes of tilting and stretching of vortex filaments?

In the context of the turbulent-spot structure, which they observed, Schubauer and Klebanoff (1955) noted that the lag in the speed of the spot at the surface may have been due to the turbulence having to propagate inwards from its place of origin, which was further out in the boundary layer. This suggests the possible relevance of Taylor diffusion, at least in a modified form for vorticity, to which we return later.

5. Singularities. If a sharp interface is a real phenomenon, then it is natural to inquire if a singularity, or near-singularity, is implied in the velocity field. If we ignore the diffusive effect of viscosity, can the equations of motion permit or encourage the development of singularities in the velocity, vorticity, and density fields? If a singularity is developed, is compressibility important? Can compressibility be important even without an effect of Mach number? This can be the case in lubrication gas films, for example.

One example of the development of singularities, where compressibility is important, involves vortex stretching and tilting (see Stuart 1998). This concerns a velocity field somewhat similar to that of (4.1) but with a dependence on $x$ as well as on $y$ and $z$ : the dependence on $x$ and $z$ is linear.

It is convenient and simpler here to use Lagrangian coordinates as in (2.7)-(2.10). If $\psi$ is the particle coordinate (in the $y$-sense) and $Y$ is the current coordinate, then for an adiabatic gas

$$
p=k \rho^{\gamma},
$$

it can be shown that $Y(\psi, t)$ satisfies

$$
Y_{t t}-C^{2}(\psi, t) Y_{\psi}^{-1-\gamma}\left[Y_{\psi \psi}-2(1-\gamma)^{-1} C^{-1} C_{\psi} Y_{\psi}\right]=0
$$

where

$$
C^{2}(\psi, t)=C_{0}^{2}(\psi)\left\{\left[1+t u_{0}(\psi)\right]\left[1+t w_{0}(\psi)\right]\right\}^{-1-\gamma} .
$$

In this expression $C_{0}(\psi)$ is the speed of sound at $t=0$, while $u_{0}(\psi)$ and $w_{0}(\psi)$ are known and are proportional to the $x$ and $z$ components of velocity at $t=0$.

In spite of the three-dimensional complexity of the present velocity field, (5.2) is almost exactly the Lagrangian equation of flow in one-dimensional gas dynamics, but there is just one significant difference. In the one-dimensional case, $C^{2}(\psi, t)$ is replaced by $C_{0}^{2}(\psi)$, a quantity that is independent of $t$. In contrast, the propagation speed in our three-dimensional case does depend on $t$ in a manner indicated by (5.3).

If the velocity component $w_{0}(\psi)$ has a negative region, as is the case in the example discussed in Sec. 4, then it can be shown that (5.2) can have a singular solution. Indeed a "similarity" structure can be constructed as the time $t$ approaches $t_{s}$, the singular time.

Writing

$$
Y=\left(t_{s}-t\right)^{\delta} g(\eta)
$$


TABLE 1. Singularity Parameter Values

\begin{tabular}{||l||l||l||l||}
\hline \hline$\gamma$ & $\delta$ & $\sigma$ & \\
\hline \hline & & & \\
\hline \hline$\infty$ & $-\frac{1}{2}$ & 0 & Incompressible \\
\hline \hline & & & \\
\hline \hline 5 & 0 & $-\frac{1}{2}$ & \\
\hline \hline & & & \\
\hline \hline 2 & $\frac{1}{2}$ & -1 & \\
\hline \hline & & & \\
\hline \hline$\frac{7}{5}$ & $\frac{3}{4}$ & $-\frac{5}{4}$ & Air \\
\hline \hline & & & \\
\hline \hline 1 & 1 & $-\frac{3}{2}$ & Isothermal \\
\hline \hline
\end{tabular}

$$
\begin{gathered}
\rho=\rho_{0}\left(t_{s}-t\right)^{\sigma} r(\eta), \\
\eta=\left(\psi-\psi_{s}\right)\left(t_{s}-t\right)^{-1 / 2},
\end{gathered}
$$

where $\psi_{s}$ is the value $\psi$ at which the singularity occurs, and ignoring higher-order terms, we find that

$$
\delta=(5-\gamma) /(2(1+\gamma)), \quad \sigma=-3 /(1+\gamma) .
$$

Table 1 indicates some typical values.

The position of the singularity and the density there depend strongly on the value of $\gamma$, the ratio of specific heats. The density always becomes singular, except in the incompressible case; in contrast the location $(Y)$ of the singularity tends to infinity as $t \rightarrow t_{s}$ if $\gamma>5$, but tends to zero if $\gamma<5$. The behaviour of the velocity and vorticity fields can also be evaluated.

6. Flow in a circular pipe. Since the pioneering work of Reynolds (1883) many scientists have studied flow in a circular pipe, both experimentally and theoretically. As mentioned earlier, Reynolds' experiments indicated the occurrence of "flashes" of turbulence in flow in a pipe; later workers, including Wygnanski and his co-workers $(1973,1975)$, have pursued this theme with some fascinating results.

From a theoretical point of view there is little firm knowledge about the instability of laminar flow: but, as mentioned earlier, it is widely believed without proof that the fully-developed laminar flow is stable to infinitesimal disturbances. However, nonlinear effects have been studied by Smith and Bodonyi (1982), who discovered by calculation an interesting neutral solution.

Wygnanski et al $(1973,1975)$ have distinguished between slugs of turbulence, which may be caused by instability of the boundary layer in the entry region of the pipe, and puffs of turbulence, which may be caused by large disturbances. They have delineated the ranges of disturbance and the Reynolds number in which the slugs and puffs occur. 
Their observations, moreover, have shown fascinating recordings from probes, the puffs and slugs having distinctive differences. For a slug there is both a sharp leading interface and a sharp trailing interface. For a puff, by way of contrast, there is a sharp trailing interface but no sharp leading interface.

The dynamics of flow near to such interfaces is incompletely understood. Are turbulent filaments or elements transported across interfaces? How is turbulence generated, or indeed destroyed, in transport through such interfaces? What is the mathematical structure of the velocity, vorticity, and pressure near to the interface? What is the role of the processes known as vortex stretching and tilting? Are singularities likely in the velocity, vorticity, and pressure fields? If so, is compressibility likely to be important at least locally?

7. A neutral mode in a circular pipe. Consider a pipe of unit radius, the radial distances within the pipe being denoted by $r$. Then laminar flow can be driven within the pipe by a pressure difference between its ends, which are far apart, provided that the Reynolds number is sufficiently small. The axial velocity is given by $\left(1-r^{2}\right)$.

If an infinitesimal perturbation is added of the form

$$
u(r) \exp [i \alpha(x-c t)+i N \theta]
$$

where $x$ is the axial coordinate, $\theta$ is the azimuthal coordinate, $t$ is the time, $\alpha$ is a wave number, $c$ is a wave speed, and $N$ is an integral azimuthal wave number, then it is known that there may be a singularity at the value of $r$ for which $1-r^{2}=c$. The singularity can be smoothed out by viscosity, or by nonlinearity. The paper of Benney and Bergeron (1969) is of great importance for its recognition and treatment of the latter case.

Smith and Bodonyi (1982) applied the ideas of Benney and Bergeron (1969) to flow in a pipe and found that they could construct a nonlinear neutral solution of the type of (7.1), neither growing nor decaying that is, provided that

$$
N=1 \quad \text { and } \quad 0.284<c<1 .
$$

They found that $c$ approaches the value 0.284 as the amplitude of (7.1) shrinks, but that $c$ approaches 1 as the amplitude becomes relatively large.

But the Smith-Bodonyi solution has no leading or trailing interfaces. Could they be fitted in some way? Are there neighbouring time-dependent solutions? These questions remain to be answered.

8. Taylor diffusion of vorticity. From the work of G. I. Taylor $(1953,1954)$ we know that a contaminant in a very long pipe is subject to an effective longitudinal diffusion process, which is brought about by a balance of longitudinal convection with radial diffusion. Thus for laminar flow in a pipe, with a velocity distribution given by $\left(1-r^{2}\right)$ for a pipe of radius unity, a contaminant $C(r, t)$ satisfies approximately the equation (Taylor, 1953)

$$
\frac{\partial C}{\partial t}-\left(D+\frac{U_{0}^{2} a^{2}}{192 D}\right) \frac{\partial^{2} C}{\partial Z^{2}}=0,
$$

provided that variations are slow in both time and space. 
Here

$$
Z=x-\frac{1}{2} U_{0} t
$$

where $x$ is the dimensional axial coordinate, $t$ is the dimensional time, $U_{0}$ is the maximum flow velocity, $a$ is the pipe radius and $D$ is the molecular diffusivity of the contaminant. The coordinate $Z$ represents translation with the average flow velocity.

If

$$
\left(\frac{U_{0} a}{D}\right)^{2}>192
$$

then the Taylor diffusion term $\left(U_{0}^{2} a^{2} / 192 D\right)$ is more important than that of molecular diffusion $(D)$. Moreover, we note that $D$ occurs in an inverted way in the Taylor diffusion term! This is a consequence of longitudinal convection allied to radial diffusion.

As Taylor (1954) remarked, a turbulent velocity profile would be required in many practical situations together with an eddy (turbulent) diffusivity to replace $D$. The numerical coefficients in (8.1), (8.2), and (8.3) would be changed, but not the structure.

We now ask the question as to whether similar processes can occur with reference to vorticity perturbations to flow in a pipe. Can such a perturbation be treated as a contaminant?

For simplicity we consider (Stuart 1981, 1996) a swirl superimposed on the basic axial flow. The circulation $\Omega$, which is the product of the local radius with the swirl velocity, is a suitable flow quantity. If we linearize the equation for the circulation and apply an analysis similar to that for contaminants (as in Taylor's case) we can show that the circulation is of the form

$$
r J_{1}\left(\lambda_{j} r\right) e^{-\lambda_{j}^{2} \nu t / a^{2}} S\left(\lambda_{j}, t\right)
$$

where $S$ satisfies

$$
\frac{\partial S}{\partial t}-\left(\nu+\frac{U_{0}^{2} a^{2}}{15 \nu \lambda_{j}^{4}}\left(8-\frac{1}{3} \lambda_{j}^{2}\right)\right) \frac{\partial^{2} S}{\partial Z^{2}}=0 .
$$

Here

$$
Z=x-\frac{2}{3} U_{0} t
$$

where $\nu$ is the kinematic viscosity, $J_{1}$ is the Bessel Function of first order, and $\lambda_{j}$ $(j=1,2,3, \ldots, n, \ldots)$ is a zero of $J_{1}$. A summation over $j$ in (8.4) would enable an initial condition to be satisfied.

The Taylor diffusion term, which is given by

$$
K_{T}=\frac{U_{0}^{2} a^{2}}{15 \nu \lambda_{j}^{4}}\left(8-\frac{1}{3} \lambda_{j}^{2}\right),
$$

is negative if $j \geq 2$. Then the effective diffusion coefficient, which is

$$
K=\nu+K_{T}
$$

changes sign at a critical value of the Reynolds number. For example if $j=2$,

$$
K<0 \quad \text { if } \quad R>66 .
$$


This negative diffusion implies a focussing rather than the usual diffusive attenuation. However, we should note that although the circulation (8.4) decays, the focussing could provide a mechanism for the promoting of interfaces between turbulent and non-turbulent regions.

This analysis has deficiencies: changes are slow, and nonlinearity is ignored. It would be of interest to determine if the Smith-Bodonyi neutral solution of Sec. 7 could be treated by such techniques, so as to produce interfaces.

9. Conclusions. In this lecture the author has attempted to expose some of the theoretical problems associated with spots, puffs, and slugs of turbulence and especially with interfaces between turbulent and non-turbulent regions of flow.

No solutions have been given! But the role (i) of vortex dynamics, especially stretching and tilting of vorticity, has been discussed. Also the role (ii) of singularities in Euler (inviscid) fields has received attention, together with (iii) the concomitant local effects of compressibility. Moreover (iv) some attention has been given to Taylor diffusion, as it might apply to a kinematical property such as vorticity; in that connection a negative diffusivity may be relevant.

Needless to say, much more mathematical research is needed. So much is abundantly clear.

The author is grateful to Professors W. Freiberger, C. Dafermos, and D. Mumford, and indeed to Brown University, for inviting him to participate in the 50th anniversary celebrations of the Division of Applied Mathematics.

\section{REFERENCES}

[1] D. J. Benney and R. F. Bergeron, A new class of nonlinear waves in parallel flows, Studies Appl. Math. 48, 181-204 (1969)

[2] B. Cantwell, D. Coles, and P. Dimotakis, Structure and entrainment in the plane of symmetry of a turbulent spot, J. Fluid Mech. 87, 641-672 (1978)

[3] V. W. Ekman, On the change from laminar to turbulent motion in liquids, Ark. Mat. Astr. Fys. 6, No. $12(1910)$

[4] H. W. Emmons and A. E. Bryson, The laminar-turbulent transition in a boundary layer, Proc. 1st U.S. National Cong. Appl. Mech., 859-868 (1951)

[5] M. Gaster, The development of a two-dimensional wave packet in a growing boundary layer, Proc. Roy. Soc. A384, 317-332 (1982)

[6] D. Henningsen, P. Spalart, and J. Kim, Numerical simulations of turbulent spots in plane Poiseuille flow, Phys. Fluids 30, 2914-2917 (1987)

[7] P. S. Klebanoff, K. D. Tidstrom, and L. M. Sargent, The three-dimensional nature of boundary-layer instability, J. Fluid Mech. 12, 1-34 (1962)

[8] O. Reynolds, An experimental investigation of the circumstances which determine whether the motion of the water shall be direct or sinuous, and of the law of resistance in parallel channels, Phil. Trans. Roy. Soc. A174, 935-982 (1883)

[9] L. Rosenhead, editor, Laminar Boundary Layers, Clarendon Press, Oxford, 1963

[10] G. B. Schubauer and P. S. Klebanoff, Contributions on the mechanics of boundary layer transition, Proc. Sympos. Boundary Layer Effects in Aerodynamics, Nat. Phys. Lab. Teddington. Also Rep. Nat. Adv. Comm. Aero. Washington, No. 1289, 1955

[11] G. B. Schubauer and H. K. Skramstad, Laminar boundary layer oscillations and transition on a flat plate, Rep. Nat. Adv. Comm., Washington, No. 909. Also J. Res. Nat. Bur. Stand. Washington, 38, 251-292, and J. Aero. Sci. 14, 69-78 (1947)

[12] J. T. Stuart, Instability and transition in pipes and channels, In "Transition and Turbulence" (ed. R. E. Meyer), Academic Press, 1981, pp. 77-94 
[13] J. T. Stuart, Evolution of vorticity in flow in a pipe, Exptl. Thermal. Fluid Sci. 13, 206-210 (1996)

[14] J. T. Stuart, Singularities in three-dimensional compressible Euler flows with vorticity, Theor. Comp. Fluid Dyn. 10, 385-391 (1998)

[15] T. Tatsumi, Stability of the laminar inlet-flow prior to the formation of Poiseuille régime, I, II, J. Phys. Soc. Japan 7, 489-495, 495-502 (1952)

[16] G. I. Taylor, Dispersion of soluble matter in solvent flowing slowly through a tube, Proc. Roy. Soc. A219, 186-203 (1953)

[17] G. I. Taylor, The dispersion of matter in turbulent flow through a pipe, Proc. Roy. Soc. A223, 446-468 (1954)

[18] I. J. Wygnanski and F. H. Champagne, On transition in a pipe. Part 1. The origin of puffs and slugs and the flow in a turbulent slug, J. Fluid Mech. 59, 281-335 (1973)

[19] I. J. Wygnanski, M. Sokolov, and D. Friedman, On transition in a pipe. Part 2. The equilibrium puff, J. Fluid Mech. 69, 283-304 (1975)

[20] F. T. Smith and R. J. Bodonyi, Amplitude-dependent neutral modes in the Hagen-Poiseuille flows through a circular pipe, Proc. Roy. Soc. A384, 463-489 (1982) 